\title{
Le Registre Suisse du Cancer de l'Enfant: premier registre du cancer national
}

\author{
Claudia E. Kuehni ${ }^{a}$, \\ Nicolas $X$. von der Weid ${ }^{b}$ \\ (pour le Groupe suisse \\ d'oncologie pédiatrique GOPS *) \\ a Directrice, Registre Suisse \\ du Cancer de l'Enfant, Institut de \\ médecine sociale et préventive, \\ Université de Berne \\ b Président du GOPS, Unité \\ d'hémato-oncologie pédiatrique, \\ DMCP, CHUV Lausanne \\ * Conseil de recherche du GOPS \\ Dr R. Angst, Aarau; Dr M. Beck \\ Popovic, Lausanne; Dr E. Bruder \\ Bâle; Dr U. Caflisch, Lucerne; \\ Dr J. Greiner, St-Gall; Dr H. Hen- \\ gartner, St-Gall; PD Dr J.-M. Joseph \\ Lausanne; PD Dr N. X. von der \\ Weid, Lausanne; Prof. Dr A. Hirt, \\ Berne; Dr C. E. Kuehni, Berne; \\ Prof. Dr F. Niggli, Zurich; \\ Dr L. Nobile Buetti, Locarno; \\ PD Dr A. H. Ozsahin, Genève; \\ Prof. Dr M. Paulussen, Bâle; \\ PD Dr Damien C. Weber, Genève.
}

Conflit d'intérêts: aucun

Correspondance:

PD Dr Nicolas von der Weid

Médecin spécialiste

en pédiatrie $\mathrm{FMH}$,

spéc. hémato-oncologie

DMCP

CHUV

CH-1011 Lausanne

Tél. 0213141334

Fax 0213143332

nicolas.von-der-weid@chuv.ch

www.kinderkrebsregister.ch www.ispm.ch
Depuis juin 2007, le Registre Suisse du Cancer de l'Enfant (RSCE) bénéficie d'une autorisation générale pour les registres, attribuée par la Commission fédérale d'experts du secret professionnel en matière de recherche médicale. Le RSCE fonctionne ainsi dorénavant comme les registres cantonaux du cancer. Cette autorisation générale pour les registres permet la récolte de données non anonymisées concernant les maladies malignes de l'enfant et de l'adolescent sur le plan national provenant de différentes sources, sans qu'un consentement formel du patient ou de sa famille ne soit nécessaire au préalable. Les patients et leur famille possèdent le droit de veto et leurs médecins traitants ont l'obligation de les informer de ce droit.

\section{Pourquoi est-ce que I'enregistrement du cancer de l'enfant est important?}

Le cancer chez l'enfant est rare. Moins d' $1 \%$ de tous les cancers diagnostiqués dans notre pays le sont chez des patients âgés de moins de 15 ans; pour la Suisse il s'agit d'environ 220 nouveaux cas par année. Le spectre diagnostique, avec une prédominance de néoplasies hématologiques, de tumeurs du SNC et de tumeurs embryonnaires, est tout à fait différent de celui du cancer chez l'adulte. Malgré sa rareté, le cancer reste la deuxième cause de décès chez l'enfant et, pour les patients guéris, les séquelles à long terme sont une cause importante de maladies chroniques et entraînent souvent une réduction de la qualité de vie.

Il est donc important que des données précises, cliniques et épidémiologiques des cancers de l'enfant soient enregistrées de façon centrale et régulièrement évaluées. Cette stratégie permet un large échange d'expertises entre les médecins traitant ces maladies et représente une condition fondamentale pour des progrès dans leur prévention et leur traitement.

C'est pourquoi les oncologues-pédiatres ont commencé dans les années 70 à enregistrer un maximum d'enfants dans des protocoles d'études cliniques internationaux visant à l'optimisation du traitement. Grâce à cette collaboration scientifique internationale, le taux de guérison du cancer de l'enfant s'est drastiquement amélioré pour atteindre actuellement environ 80\%. Pour assurer une surveillance exhaustive, la recherche étiologique et l'optimisation des traitements, il est toutefois nécessaire d'analyser également les données d'enfants et d'adolescents qui, pour des raisons diverses, n'ont pas pu être inclus dans des études cliniques internationales.

C'est pourquoi des registres nationaux spécifiques du cancer de l'enfant ont été développés dans de nombreux pays, par exemple en Allemagne (www.kinderkrebsregister.de) et au Royaume-Uni (www.ccrg.ox.ac.uk). Le RSCE (www.kinderkrebsregister.ch) a été fondé en 1976 par le Groupe suisse d'oncologie pédiatrique (GOPS). Initialement, seules les données de patients officiellement traités dans une étude clinique étaient enregistrées; depuis 1981 les données des patients hors études ont également été récoltées. Depuis le début des années 90, le suivi à long terme avec enregistrement des effets indésirables tardifs a été introduit.

\section{Responsabilité du Registre National du Cancer de I'Enfant}

Le RSCE est conduit en commun par le Groupe suisse d'oncologie pédiatrique (GOPS) (www. spog.ch) et l'Institut de médecine sociale et préventive de l'Université de Berne (www.ispm.ch), où il est établi depuis 2004.

\section{Buts, critères d'inclusion et méthodes du RSCE}

Le RSCE enregistre des données sur le diagnostic, la présentation clinique, le traitement et le pronostic de toutes les maladies malignes de l'enfant et de l'adolescent, avec les buts suivants:

1. fournir des données nationales représentatives de l'incidence, du taux de survie, des tendances chronologiques et de la répartition régionale;

2. participer à la recherche de facteurs de risque génétiques et environnementaux du cancer de l'enfant et au développement de concepts préventifs; 
3. enregistrer des informations valables sur le pronostic à long terme et les effets indésirables tardifs du traitement et développer des stratégies pour éviter ou minimiser ces séquelles à long terme;

4. fournir des données fiables pour la planification et le contrôle de qualité de la prise en charge médicale des enfants et des adolescents souffrant d'affections malignes en Suisse.

\section{Critères d'inclusion}

Le RSCE enregistre tous les cas de tumeurs solides malignes, de leucémies et lymphomes, de tumeurs du SNC (y inclus les tumeurs bénignes) et les histiocytoses à cellules de Langerhans ou autres chez les enfants et les adolescents (jusqu'à 20 ans, qui habitent en Suisse ou y sont traités). Les tumeurs sont codées d'après la classification internationale pour les maladies oncologiques, $3^{\mathrm{e}}$ révision (ICD-O-3), et la classification internationale des cancers de l'enfant, $3^{\text {e }}$ révision (ICCC-3) [1].

\section{Données récoltées}

Le RSCE récolte des données démographiques, noms et adresses au moment du diagnostic, des informations importantes sur l'anamnèse familiale et les affections préexistantes, les données diagnostiques (diagnostic exact, localisation et morphologie de la tumeur, stade, métastases) ainsi que des données détaillées sur le traitement et l'évolution, les modalités de traitement, l'inclusion dans une étude clinique, les données concernant la rémission et les récidives, les effets à long terme, les tumeurs secondaires et les décès.

\section{D'un registre clinique des tumeurs au Registre Suisse du Cancer de I'Enfant}

Jusqu'à présent, le RSCE collectait ses données exclusivement au travers des neuf Unités d'hémato-oncologie pédiatriques (Aarau, Bâle, Berne, Genève, Lausanne, Lucerne, St-Gall, Tessin, Zurich). Cette stratégie était basée sur l'a priori que pratiquement tous les patients atteints de cancer en Suisse étaient traités dans l'une de ces neuf unités. Tous ces centres disposent d'une équipe spécialisée multidisciplinaire et remplissent toutes les conditions pour une prise en charge optimale de ces patients, y inclus l'enregistrement dans des études d'optimisation thérapeutique internationales.

Une comparaison exécutée récemment (2006-2007) des données du RSCE avec celles des Registres cantonaux du cancer (dans les cantons qui en possèdent un) a toutefois montré que seuls environ $85 \%$ des enfants cancéreux de ces cantons étaient enregistrés dans le RSCE. Pour obtenir l'enregistrement réellement exhaustif de tous les patients pédiatriques, le RSCE doit dès maintenant collecter des données provenant de différentes sources. Ceci veut dire par exemple des données provenant d'autres hôpitaux, des rapports de pathologie, de cytologie et des échanges réguliers avec les Registres cantonaux du cancer.

\section{Aspects de protection des données}

Depuis 2004, le RSCE possède une autorisation spéciale de la Commission fédérale d'experts du secret professionnel en matière de recherche médicale. En juin 2007, cette autorisation spéciale est devenue une autorisation générale pour la tenue d'un registre.

La Commission fédérale d'experts existe depuis l'application de la Loi suisse sur la protection des données, article 321bis, en 1993 et attribue des autorisations pour des projets de recherche, des hôpitaux, des instituts ainsi que des registres médicaux. D'après cette loi, en principe seules les données cliniques anonymisées ou après le recueil préalable du consentement du patient peuvent être transmises et utilisées par un registre. Si ceci n'est pas possible, on peut demander une autorisation spéciale à la Commission fédérale d'experts.

Les conditions pour l'attribution d'une telle autorisation sont:

1. que les patients aient été informés de leur droit de refuser l'utilisation de leurs données à des buts de recherche (droit de veto) et qu'ils n'ont pas expressément refusé cette utilisation;

2. que la recherche n'est pas possible avec des données anonymisées. Ceci peut avoir différentes raisons (par exemple empêcher des enregistrements double, enregistrer des données dans le futur, pour lesquelles l'identité du patient doit être connue);

3. qu'il est impossible ou extrêmement difficile d'obtenir le consentement du patient (p.ex. pour des patients décédés ou qui ont déménagé);

4. que l'intérêt de la recherche dépasse clairement les intérêts de la conservation du secret pour les patients. Ceci nécessite une appréciation du conflit d'intérêts qui existe entre le maintien du secret et de la confidentialité pour les patients d'une part et les intérêts généraux du projet de recherche concerné qui peut par exemple nécessiter un grand nombre de patients, améliorer les possibilités thérapeutiques ou de façon plus générale le progrès dans le domaine médical. 
Ces conditions sont clairement remplies par les registres du cancer basés sur la population, et les registres cantonaux du cancer possèdent une telle autorisation de registre. Leurs expériences avec cette procédure sont excellentes. Il n'arrive que très rarement que des patients souhaitent que leurs données ne soient pas enregistrées.

Depuis le 25 juillet 2007, le RSCE possède une telle autorisation de registre et est autorisé à enregistrer des données personnelles non anonymisées d'enfants et d'adolescents malades d'un cancer et habitant ou étant traités en Suisse. Cette autorisation est également donnée à tous les médecins en pratique privée ou hospitalière, leur personnel auxiliaire, les instituts de pathologie, les laboratoires médicaux et les institutions en Suisse qui collectent des données statistiques (p.ex. l'OFS). Ceci permet à ces personnes et institutions de transmettre les données de leurs patients sans porter atteinte au secret professionnel.

Cette autorisation générale demande comme condition sine qua non que des mesures strictes de protection des données soient tenues. Cellesci comportent la conservation de tous les documents dans les locaux fermés, un secret de fonction absolu de tous les collaborateurs du RSCE, la conduite de la base de données sur un serveur spécifique hautement sécurisé et un enregistrement séparé avec accès restreint aux données personnelles. Pour l'évaluation et l'analyse, les données individuelles ne seront transmises aux universités, instituts scientifiques ou établissements similaires que sous une forme anonymisée.

\section{Devoir d'information des médecins traitants}

Les personnes dont les données sont transmises au RSCE doivent être informées qu'elles ont le droit de s'opposer à la transmission et l'utilisation de leurs données (information et droit de veto). Le devoir d'information concerne essentiellement les médecins traitants. Ceux-ci sont en contact direct avec le patient et peuvent ainsi prendre connaissance de leur refus de mettre à disposition leurs données personnelles; ils doivent alors s'assurer que ce veto est respecté. L'information aux patients et leurs représentants légaux peut se faire sous une forme relativement générale, par exemple une brochure d'information aux patients, une affiche dans le cabinet médical ou à l'hôpital ou un feuillet d'information. Si un patient ou son représentant légal fait usage de son droit de veto, le médecin traitant est obligé de l'annoncer au RSCE. Ainsi, d'autres données concernant ce patient et provenant d'autres sources (par exemple des rapports de laboratoire) pourront être anonymisées.

\section{Informations complémentaires et matériels pour l'information aux patients}

Des informations détaillées concernant la méthodologie et les résultats du RSCE peuvent être consultés sur le site web du RSCE (www.kinder krebsregister.ch), ainsi que dans les rapports annuels [2-3] et les publications [4-7].

L'autorisation de la Commission fédérale d'experts du secret professionnel en matière de recherche médicale peut être consultée sur le site web du RSCE (www.kinderkrebsregister.ch), le site web de la Société suisse de pédiatrie (www.swiss-paediatrics.org) et le site web du GOPS (www.spog.ch). Le site web de la Commission fédérale d'experts de l'Office fédéral de la santé publique contient des informations détaillées sur ces autorisations (www.bag.admin.ch/ org/02329/index.html?lang=fr).

Nous avons préparé deux modèles pour l'information aux patients qui peuvent être utilisés par les médecins traitants: une petite affiche pour accrocher dans le cabinet médical ou la clinique ainsi qu'un texte court à introduire dans des brochures d'informations aux patients. Ces instruments peuvent également être téléchargés des homepages suivantes: RSCE (www.kinder krebsregister.ch), Société suisse de pédiatrie (www.swiss-paediatrics.org) et GOPS (www. spog.ch).

\section{Références}

1 Steliarova-Foucher E, Stiller C, Lacour B, Kaatsch P. International Classification of Childhood Cancer. Third edition. Cancer. 2005;103:1457-67.

2 The Swiss Childhood Cancer Registry. Annual Report 2004. www.kinderkrebsregister.ch/Daten/ SKKR_AnnualReport_2004.pdf.

3 The Swiss Childhood Cancer Registry. Annual Report 2005/2006. www.kinderkrebsregister.ch/ Daten/SKKR_AnnualReport_2005_2006.pdf.

4 Michel G, von der Weid N, Zwahlen M, Adam M, Rebholz CE, Kuehni CE. The Swiss Childhood Cancer Registry: rationale, organisation and results for the years 2001-2005. Swiss Med Wkly. 2007; 137:502-9.

5 Michel G, von der Weid N, Zwahlen M, Redmond S, Strippoli MPF, Kuehni CE. Incidence of childhood cancer in Switzerland: the Swiss Childhood Cancer Registry. Pediatr Blood Cancer. 2008;50:46-51.

6 von der Weid N. Late effects in long-term survivors of ALL in childhood: Experiences from the SPOG late effects study. Swiss Med Wkly. 2001;131:180-7.

7 von der Weid N, Mosimann I, Hirt A, Wacker P, Nenadov Beck M, Imbach P, et al. Intellectual outcome in children and adolescents with acute lymphoblastic leukaemia treated with chemotherapy alone: age- and sex-related differences. Eur J Cancer. 2003;39(3):359-65. 